Pacific Journal of Mathematics

SCHUR INDICES OVER THE 2-ADIC FIELD 


\section{SCHUR INDICES OVER THE 2-ADIC FIELD}

\section{TOSHIHIKo YAMADA}

In this paper it is proved that if $G$ is a finite group with abelian Sylow 2-subgroups, then the Schur index of any character of $G$ over the 2-adic numbers $Q_{2}$ is equal to 1 . Examples are given so as to show that this statement is false for each odd prime $p$.

The problem of determining the Schur index of a character of a finite group was reduced by $R$. Brauer and $E$. Witt to the case of handling hyper-elementary groups at $q, q$ being a prime. Each of these groups has a cyclic normal subgroup with a factor group which is a $q$-group. Let $p$ be a prime and $Q_{p}$ the $p$-adic numbers. Let $G$ be a hyper-elementary group at $q$ and $\chi$ an irreducible character of $G$. It follows from a result of Witt [1] that if $p=$ $q \neq 2$ then the Schur index $m_{Q_{p}}(\chi)$ of $\chi$ over $Q_{p}$ is equal to 1 . This statement is false for the case $p=q=2$, because the quaternion group of order $2^{3}$ has an irreducible character $\chi$ with $m_{Q_{2}}(\chi)=2$.

The purpose of this paper is to show that the above statement also holds for the case $p=q=2$, provided the Sylow 2-subgroups of a hyper-elementary group at 2 are abelian. In fact, we will prove more generally the following theorem.

THEOREM. Let $G$ be a finite group with abelian Sylow 2-subgroups. Let $\chi$ be any irreducible character of $G$. Then $m_{Q_{2}}(\chi)=1$, that is the Schur index of $\chi$ over the 2-adic numbers $Q_{2}$ is equal to 1.

Proof. It is well-known that $m_{Q_{2}}(\chi)=1$ or 2 (cf. [1]), so $m_{Q_{2}}(\chi)$ equals its 2-part. Let $n$ be the exponent of $G$ and let $L$ be the subfield of $Q_{2}\left(\zeta_{n}\right), \zeta_{n}$ a primitive $n$th root of unity, such that $L \supset Q_{2}(\chi), 2 \nmid\left[L: Q_{2}(\chi)\right]$ and $\left[Q_{2}\left(\zeta_{n}\right): L\right]$ is a power of 2 . By the Brauer-Witt theorem [3, p. 31] there is an $L$-elementary subgroup $H$ of $G$ with respect to 2 and an irreducible character $\theta$ of $H$ with the following properties: (1) there is a normal subgroup $N$ of $H$ and a linear character $\psi$ of $N$ such that $\theta=\psi^{H} ;(2) H / N \cong \operatorname{Gal}(L(\psi) / L)$, in particular, $H / N$ is a 2-group; (3) $L(\theta)=L$; (4) $m_{L}(\theta)=m_{L}(\chi)=$ $m_{Q_{2}(\chi)}(\chi)=m_{Q_{2}}(\chi) ;(5)$ for every $h \in H$ there is a $\tau(h) \in \operatorname{Gal}(L(\psi) / L)$ such that $\psi\left(h n h^{-1}\right)=\tau(h)(\psi(n))$ for all $n \in N$; (6) $m_{L}(\theta)$ is the index of the crossed product $(\beta, L(\psi) / L)$ where, if $D$ is a complete set of coset representatives of $N$ in $H(1 \in D)$ with $h h^{\prime}=n\left(h, h^{\prime}\right) h^{\prime \prime}$ for $h, h^{\prime}, h^{\prime \prime} \in D, n\left(h, h^{\prime}\right) \in N$, then $\beta\left(\tau(h), \tau\left(h^{\prime}\right)\right)=\psi\left(n\left(h, h^{\prime}\right)\right)$. Since $\psi$ is 
a linear character of $N$, the values of the factor set $\beta$ are roots of unity.

Denote by $N_{0}$ the kernel of $\psi$. Then the factor group $N / N_{0}$ is cyclic. Put $2^{r} t=\left|N / N_{0}\right|,(2, t)=1$. It is easy to see that there exist elements $a, b$ of $N$ such that $N / N_{0}=\left\langle a N_{0}\right\rangle \times\left\langle b N_{0}\right\rangle, a^{2 r} \in N_{0}$, $b^{t} \in N_{0}$ and that the order of $a$ is a power of 2 . We have $\psi(a)=\zeta_{2^{r}}$, $\psi(b)=\zeta_{t}$, and $Q_{2}(\psi)=Q_{2}\left(\zeta_{2} r, \zeta_{t}\right)$, where $\zeta_{2 r}$ and $\zeta_{t}$ are some primitive $2^{r}$ th and $t$ th roots of unity, respectively. Let $P$ be a Sylow 2-subgroup of $H$, which contains $a$. Since $H / N$ is a 2-group, we may clearly assume that $D \subset P$. By assumption, $P$ is abelian. Hence for each $x \in D, x a x^{-1}=a$, and so

$$
\theta(a)=\psi^{H}(a)=\sum_{x \in D} \psi\left(x a x^{-1}\right)=|D| \psi(a)=|D| \zeta_{2^{r}}
$$

Consequently, $\zeta_{2} \in L=L(\theta)$.

Since $L(\psi)=L\left(\zeta_{2} r, \zeta_{t}\right)=L\left(\zeta_{t}\right),(2, t)=1$, it follows that the extension $L(\psi) / L$ is unramified. Recall that the values of the factor set $\beta$ are roots of unity. Hence the crossed product $(\beta, L(\psi) / L)$ is similar to $L$, i.e., $(\beta, L(\psi) / L) \sim L$ (cf. [3, Lemma 4.2]). This implies $m_{Q_{2}}(\chi)=m_{L}(\theta)=1$, and the theorem is proved.

If $p$ is an odd prime, then Witt [1] determined that $m_{Q_{p}}(\chi)$ divides $p-1$ for an irreducible character $\chi$ of a finite group $G$. Let $d$ be a natural number that divides $p-1$. We now give an irreducible character $\chi$ of a finite group $G$ with abelian Sylow $p$-subgroups such that $m_{Q_{p}}(\chi)=d$ : The group $G$ is generated by the elements $x, y$ with defining relations

$$
x^{p}=1, \quad y^{d(p-1)}=1, \quad x y x^{-1}=x^{r},
$$

where $r$ is a primitive root modulo $p$. (This group was dealt with in Appendix of [2].)

Now put $H=\langle x\rangle \times\left\langle y^{p-1}\right\rangle$. Then $H$ is a normal, cyclic subgroup of $G$ of order $p d$, the factor group $G / H$ is cyclic of order $p-1$, and $G=H \cup H y \cup \cdots \cup H y^{p-2}$. Let $\psi$ be the faithful linear character of $H$ given by $\psi(x)=\zeta_{p}, \psi\left(y^{p-1}\right)=\zeta_{d}$. For each $i=1, \cdots$, $p-2$, the character $\psi^{y^{i}}$ of $H$ defined by $\psi^{y^{i}}(z)=\psi\left(y^{i} z y^{-i}\right), z \in H$, is algebraically conjugate to $\psi$ over the field $Q_{p}\left(\zeta_{d}\right)$, and $\psi^{\nu^{i}} \neq \psi$. It follows that the induced character $\chi=\psi^{G}$ is irreducible and that the simple component of the group algebra $Q_{p}[G]$ which corresponds to $\chi$ is isomorphic to the cyclic algebra $B=\left(\zeta_{d}, Q_{p}\left(\zeta_{d}, \zeta_{p}\right) / Q_{p}\left(\zeta_{d}\right), \sigma\right)$, where $\langle\sigma\rangle=\operatorname{Gal}\left(Q_{p}\left(\zeta_{d}, \zeta_{p}\right) / Q_{p}\left(\zeta_{d}\right)\right), \sigma\left(\zeta_{p}\right)=\zeta_{p}^{r}, \sigma\left(\zeta_{d}\right)=\zeta_{d}$ (cf. Propositions $3.4,3.5$ of [3]). Since $p \equiv 1(\bmod d)$, then $Q_{p}\left(\zeta_{d}\right)=Q_{p}$, so $B=$ $\left(\zeta_{d}, Q_{p}\left(\zeta_{p}\right) / Q_{p}, \sigma\right)$. It is easy to see that the index of this cyclic algebra is equal to $d$ (see also Theorem 4.3 of [3]). Thus we conclude that $m_{Q_{p}}(\chi)=d$. 
The above example shows that the similar statement to the theorem for each odd prime $p$ does not hold.

\section{REFERENCES}

1. E. Witt, Die algebraische Struktur des Gruppenringes einer endlichen Gruppe über einem Zahlkörper, J. Reine Angew. Math., 190 (1952), 231-245.

2. T. Yamada, On the group algebras of metacyclic groups over algebraic number fields, J. Fac. Sci. Univ. Tokyo, 15 (1968), 179-199.

3. - The Schur Subgroup of the Brauer Group, Lecture Notes in Mathematics, Vol. 397, Springer-Verlag, 1974.

Received May 27, 1977.

TOKYo Metropolitan University

TOKYO, JAPAN 



\section{PACIFIC JOURNAL OF MATHEMATICS}

\section{EDITORS}

RICHARD ARENS (Managing Editor)

University of California

Los Angeles, California 90024

C. W. Curtis

University of Oregon

Eugene, OR 97403

C. C. MOORE

University of California

Berkeley, CA 94720

\section{J. DUGUNDJI}

Department of Mathematics University of Southern California Los Angeles, California 90007

R. Finn AND J. Milgram Stanford University Stanford, California 94305

ASSOCIATE EDITORS
E. F. BECKENBACH

B. H. NeUMANN

F. WOLF

K. YoshidA

\section{SUPPORTING INSTITUTIONS}

UNIVERSITY OF BRITISH COLUMBIA UNIVERSITY OF SOUTHERN CALIFORNIA CALIFORNIA INSTITUTE OF TECHNOLOGY STANFORD UNIVERSITY UNIVERSITY OF CALIFORNIA MONTANA STATE UNIVERSITY UNIVERSITY OF TOKYO UNIVERSITY OF NEVADA, RENO UNIVERSITY OF UTAH NEW MEXICO STATE UNIVERSITY OREGON STATE UNIVERSITY UNIVERSITY OF OREGON

WASHINGTON STATE UNIVERSITY UNIVERSITY OF WASHINGTON OSAKA UNIVERSITY 


\section{Pacific Journal of Mathematics}

\section{Vol. 74, No. $1 \quad$ May, 1978}

Gerald Arthur Anderson, Computation of the surgery obstruction groups

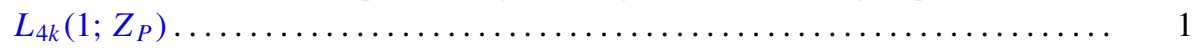

R. K. Beatson, The degree of monotone approximation ................ 5

Sterling K. Berberian, The character space of the algebra of regulated functions . . . 15

Douglas Michael Campbell and Jack Wayne Lamoreaux, Continua in the plane with

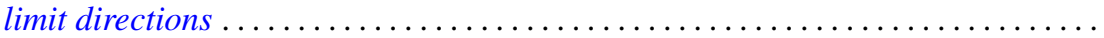

R. J. Duffin, Algorithms for localizing roots of a polynomial and the Pisot

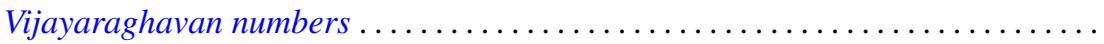

Alessandro Figà-Talamanca and Massimo A. Picardello, Functions that operate on

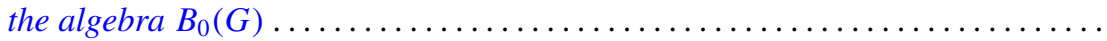

John Erik Fornaess, Biholomorphic mappings between weakly pseudoconvex

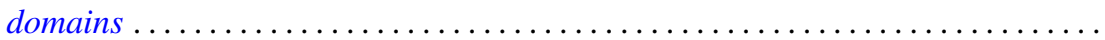

Andrzej Granas, Ronald Bernard Guenther and John Walter Lee, On a theorem of S.

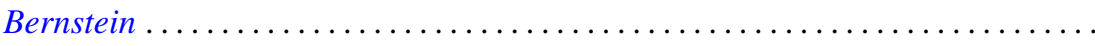

Jerry Grossman, On groups with specified lower central series quotients . .........

William H. Julian, Ray Mines, III and Fred Richman, Algebraic numbers, a constructive development . . . . . . . . . . . . . . . . . . . . . . .

Surjit Singh Khurana, A note on Radon-Nikodým theorem for finitely additive

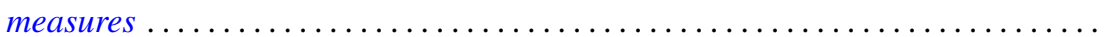

Garo K. Kiremidjian, A Nash-Moser-type implicit function theorem and nonlinear

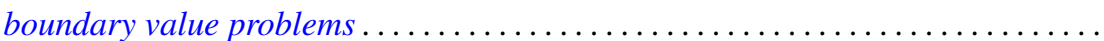

Ronald Jacob Leach, Coefficient estimates for certain multivalent functions ....

John Alan MacBain, Local and global bifurcation from normal eigenvalues. II . . 133

James A. MacDougall and Lowell G. Sweet, Three dimensional homogeneous algebras...

John Rowlay Martin, Fixed point sets of Peano continua ......

R. Daniel Mauldin, The boundedness of the Cantor-Bendixson order of some analytic sets...

Richard C. Metzler, Uniqueness of extensions of positive linear functions ..

Rodney V. Nillsen, Moment sequences obtained from restricted powers . .

Keiji Nishioka, Transcendental constants over the coefficient fields in differential elliptic function fields...

Gabriel Michael Miller Obi, An algebraic closed graph theorem

Richard Cranston Randell, Quotients of complete intersections by $\mathbf{C}^{*}$ actions . . 221

Bruce Reznick, Banach spaces which satisfy linear identities . .

Bennett Setzer, Elliptic curves over complex quadratic fields...

Arne Stray, A scheme for approximating bounded analytic functions on certain subsets of the unit disc.

Nicholas Th. Varopoulos, A remark on functions of bounded mean oscillation and bounded harmonic functions. Addendum to: "BMO functions and the

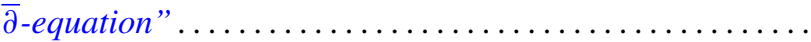

Charles Irvin Vinsonhaler, Torsion free abelian groups quasi-projective over their

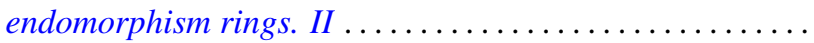

Thomas R. Wolf, Characters of $p^{\prime}$-degree in solvable groups ... 